\title{
Salivary responding and the duration of measurement
}

\author{
LEONARD H. EPSTEIN \\ University at Buffalo, Buffalo, New York \\ and \\ LUCENE WISNIEWSKI, PETRINA DEFLAVIA, and SHARI MITCHELL \\ University of Pittsburgh, Pittsburgh, Pennsylvania
}

\begin{abstract}
The Strongin-Hinsie Peck whole-mouth salivation measure (Peck, 1959) is typically collected for a 2 -min duration. This study compared saliva collected for $120 \mathrm{sec}$ with saliva collected for shorter durations ( 30 and $60 \mathrm{sec}$ ) over repeated presentation of gustatory cues. Results showed reliable increases in salivation from a water stimulus baseline to the first presentation of lemon juice as a function of measurement duration. Repeated measures analysis of variance showed overall decreases in salivation across each measurement duration, with a greater rate of habituation for the 120-sec interval than for the 30 - and 60-sec intervals. These data suggest that shorter measurement intervals can be used to measure salivation in acute and repeated measurement paradigms, but the change in response to repeated stimulus presentations is more pronounced for the longer measurement duration.
\end{abstract}

Salivation is an important physiological measure in the study of intake and ingestive behaviors. Salivation has been shown to change in response to olfactory and gustatory cues as a function of factors that initiate intake, such as the degree of hunger and deprivation (Wardle, 1987; Wooley \& Wooley, 1973) and the palatability of the food (Klajner, Herman, Polivy, \& Chabra, 1981; Wooley \& Wooley, 1973). Salivation may also be useful in understanding mechanisms for different problems that involve ingestion, such as dietary restraint (Legoff \& Spiegelman, 1987), obesity (Guy-Grand \& Goga, 1981) and eating disorders (Legoff, Leichner, \& Spiegelman, 1988).

Sensory responses are modified by the repetition of gustatory and olfactory stimulation that occurs during intake, and changes in these sensory responses may play a role in the development of satiety. Both animal (Rolls, Murzi, Yaxley, Thorpe, \& Simpson, 1986; Smithers-Mulvey \& Hall, 1992) and human (Epstein, Caggiula, Perkins, Mitchell, \& Rodefer, 1992; Epstein, Mitchell, \& Caggiula, 1993; Epstein, Rodefer, Wisniewski, \& Caggiula, 1992; Wisniewski, Epstein, \& Caggiula, 1992) research has shown habituation, dishabituation, and recovery of behavioral, subjective, and physiological responses to food. Habituation of salivation is related to satiety (Epstein, Caggiula, et al., 1992; Wisniewski et al., 1992), whereas dishabituation or recovery of salivation is related to an increase in intake (Wisniewski et al., 1992).

There is a wide variety of methods to assess salivation, with one of the most popular being the whole-mouth swab

Appreciation is expressed to Mark Kristal, Michelle Myers, and Rocco Paluch for comments on earlier versions of this manuscript. Reprints can be obtained from L. H. Epstein, Department of Psychology, University at Buffalo, Park Hall, Buffalo, NY 14260. technique (Peck, 1959). This method involves collecting saliva in dental rolls at three sites over a 2 -min period. The results correlate strongly with those using other wholemouth methods (Navaresh \& Christenen, 1982). Furthermore, swab techniques such as this correlate well with direct measurement of parotid salivation (Pangborn, Witherly, \& Jones, 1979; White, 1977).

We have been using the whole-mouth method in our habituation and satiety research, using 2-min measurement duration and 2-min intertrial intervals over 8-10 presentations of gustatory and/or olfactory cues (Epstein, Caggiula, et al., 1992; Epstein, Caggiula, Rodefer, Wisniewski, \& Mitchell, 1993; Epstein, Mitchell, \& Caggiula, 1993; Epstein, Rodefer, et al., 1992; Wisniewski et al., 1992). This protocol requires up to $40 \mathrm{~min}$ to complete 10 trials, which is longer than the duration of most meals. In order to model food presentation in intervals that are more related to typical meals, it may be necessary to use shorter measurement duration. Although investigators have used shorter (Staats \& Hammond, 1972) and longer (Klajner et al., 1981) measurement durations, there are no data available on possible differences in salivation response as a function of duration of saliva collection. This methodological study is designed to compare the amount of salivation and the pattern of salivation responses collected during three different measurement durations $(30,60$, and $120 \mathrm{sec})$ in response to 10 repeated presentations of a gustatory stimulus.

\section{METHOD}

\section{Subjects}

Eighteen women, 18-35 (19.9 \pm 1.4$)$ years old, recruited through an advertisement in the University of Pittsburgh's student newspaper were tested. The subjects were excluded if they were currently dieting, had dietary restraint scores (Herman, 1978) above $16(9.7 \pm 3.8)$, 
or had BMI values $(21.9 \pm 2.0)$ exceeding 28 (Must, Dallal, \& Dietz, 1991). The subjects who were currently taking medications that influence salivation (e.g.. Elavil, Benadryl, or atropine) or who reported significant health problems (e.g., diabetes, Sjogren's syndrome, cancer) were excluded. Inclusion in the study required that, at screening, the subject rate the flavor of the lemon test stimulus as at least moderately liked on a 100-point scale of liking $(69.9$ \pm 13.7 ).

\section{Procedures}

The subjects were screened by telephone to determine whether they met the inclusion criteria. They were randomly assigned to one of two groups ( $n=9$ per group) in a mixed design with one between factor (groups) and two within factors (duration of measurement $[30,60$, and $120 \mathrm{sec}]$ and trial $[1-10])$. The design of the experiment is shown in Table 1. In one group, the interval between stimulus presentations was kept at 4 min for all stimulus measurement durations, consistent with the interval between stimulus presentations when both the measurement duration and intertrial interval are $2 \mathrm{~min}$. For this group, the intertrial intervals were 210,180 , and $120 \mathrm{sec}$ for the $30-, 60-$, and $120-\mathrm{sec}$ conditions, respectively. Since varying the intertrial intervals can influence the rate of habituation (Siddle, Stephenson, \& Spinks, 1983), it was important to assess whether differences in intertrial intervals that arose from studying measurement duration affected the evaluation of duration. For the second group, the intertrial interval was kept at $2 \mathrm{~min}$, but the rate of stimulus presentation varied across conditions. The interstimulus presentation intervals were 150,180 , and $240 \mathrm{sec}$ for the $30-, 60-$, and 120 -sec conditions, respectively.

The subjects in each group had their salivation measured for 30,60 , and $120 \mathrm{sec}$ in three separate sessions scheduled for the same time each day between 1200 and $1700 \mathrm{~h}$, with 1-3 days between each session. The subjects were instructed to abstain from food and caffeinated beverages at least $4 \mathrm{~h}$ before each session. The order of duration of measurement was counterbalanced across subjects.

Upon arrival at the laboratory for the first session, the subject's height and weight were measured, the protocol was explained, and the subjects completed the consent forms. Two baseline salivary measures were obtained to presentations of $0.3 \mathrm{ml}$ of water. After the second baseline salivary measure, the subject rated the intensity of the stimulus. The baseline trials were followed by 10 trials of a habituating stimulus of $0.3 \mathrm{ml}$ of $2: 1$ lemon juice (Realemon, Borden, Inc., Columbus, $\mathrm{OH}$ ) to sugar solution. Taste stimuli were presented in the middle of the tongue. The subjects rated the intensity of the stimulus after each of the experimental trials.

\section{Measurement}

Salivary volume was measured using the Strongin-Hinsie Peck (Peck, 1959) method. In this procedure, the subject placed three 1.5-

Table 1

Experimental Design Representing Timing Across the First Two Trials

$\frac{\text { Time (in minutes) }}{n .1 .2 .3 .4 .5 .6 .7 .8}$

Constant Interstimulus Interval (in seconds)

$\begin{array}{rll}30 & |-| & |-| \\ 60 & |--| & |--| \mid \\ 120 & |-\ldots \ldots| & |--\ldots|\end{array}$

Constant Intertrial Interval (in seconds)

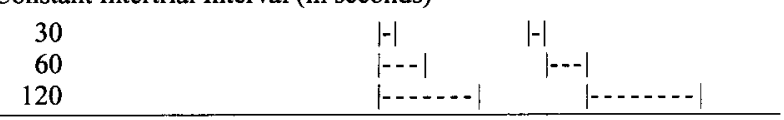

in. dental rolls (Patterson Dental Co., Minneapolis, MN) in her mouth for the collection period. The subject placed one dental roll on both the left and the right side of the mouth between the cheek and lower gum and one under the tongue. Immediately after the collection period, the subject removed the dental rolls and sealed them in a plastic bag. Salivation volume was determined by pre- and postweighings of the dental rolls to within $0.01 \mathrm{~g}$. Stimulus intensity was assessed by the subjects' ratings on a $100-\mathrm{mm}$ line anchored by not very/very.

\section{RESULTS}

A two-way mixed analysis of variance (ANOVA) on baseline salivation to the water stimulus showed significant differences by duration $[F(2,32)=4.35, p=.036]$, with $0.661,0.966$, and $1.053 \mathrm{~g}$ for the $30-, 60-$, and 120 -sec conditions, respectively. Likewise, salivation change in response to experimental stimulus presentation showed significant differences as a function of stimulus duration $[F(2,32)=$ $6.12, p=.006]$. These data are shown in Figure 1. The characteristics of a regression line relating the mean salivation change for each measurement duration to time are $y=$ $0.422+$ (time $\times 0.014 \mathrm{~g}$ ). The Pearson product-moment correlation coefficient for the regression line drawn is .96, with a standard error of estimate of .08 . These data suggest that within the measurement durations studied, there is generally a linear relationship between the amount of salivation and stimulus duration.

Salivation changes from baseline water stimulation to the repeated stimulus presentations were assessed by a three-factor mixed ANOVA with one between factor (groups) and two within factors (duration and trials). Significant main effects of trials $[F(9,144)=4.51, p<.001]$ and measurement duration $[F(9,144)=11.38, p<.001]$ were observed (see Figure 2 ). In addition, there was a marginally significant interaction between trials and duration $[F(18,144)=1.61, p=.057]$. Contrasts of differences in rate of change across groups showed a significant difference between the $30-$ and 120 -sec durations $[F(2,16)=$ $9.70, p=.002]$ and a marginally significant difference between the 30- and 60-sec durations $[F(2,16)=1.481, p=$ $.058]$, but no differences in the rate of change between 60 and $120 \mathrm{sec}(p=.257)$. There was no main effect of group and no significant interaction of groups $\times$ trials or groups $\times$ duration $\times$ trials.

To further evaluate the differences in rate of change across measurement durations, the salivation changes were regressed across the 10 trials to assess differences in slope. The 30-, 60-, and 120-sec lines had slopes of -0.027 , -0.041 , and -0.080 , respectively, consistent with the progressively greater rate of change based on measurement interval.

No differences were observed in baseline intensity as a function of stimulus duration $[F(2,32)=0.17]$, with baseline intensities for the 30-, 60-, and 120-sec durations of $9.1,10.9$, and 10.6, respectively. Likewise, no effect was observed in perceived intensity change from baseline to the first lemon stimulus as a function of stimulus duration 
$[F(2,32)=0.99]$, with measurements for the $30-, 60-$, and 120 -sec durations of $50.0,41.7$, and 42.9 , respectively. The changes in subjective intensity from baseline in Figure 3 showed no consistent pattern of change by groups over time $[F(9,144)=0.66]$, though there were some differences in the pattern of change by group $[F(18,288)=$ $1.77, p=.028]$. The intensities for the $30-\mathrm{sec}$ group slightly decreased and then increased over trials, whereas the 60 - and 120-sec groups slightly increased and then de-

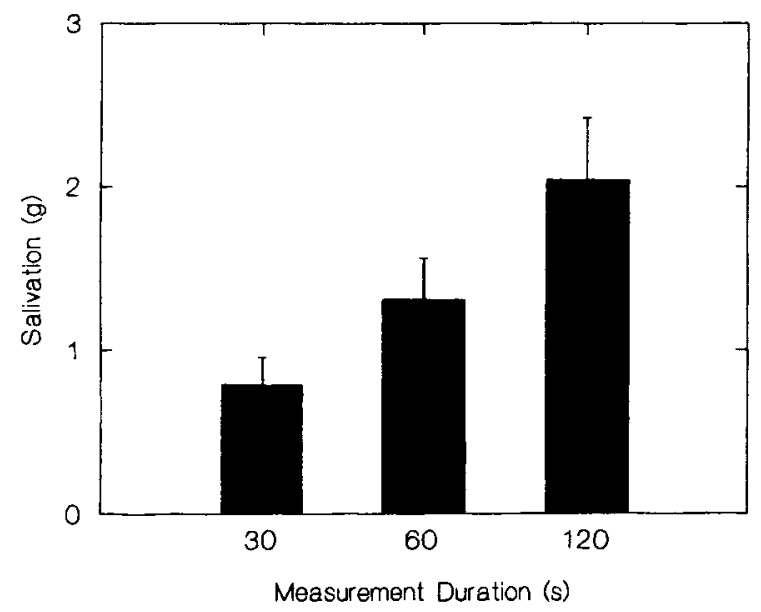

Figure 1. Mean change $( \pm S E M)$ in saliva collected from baseline for the first presentation of lemon juice for the 30-, 60-, and 120-sec measurement durations.

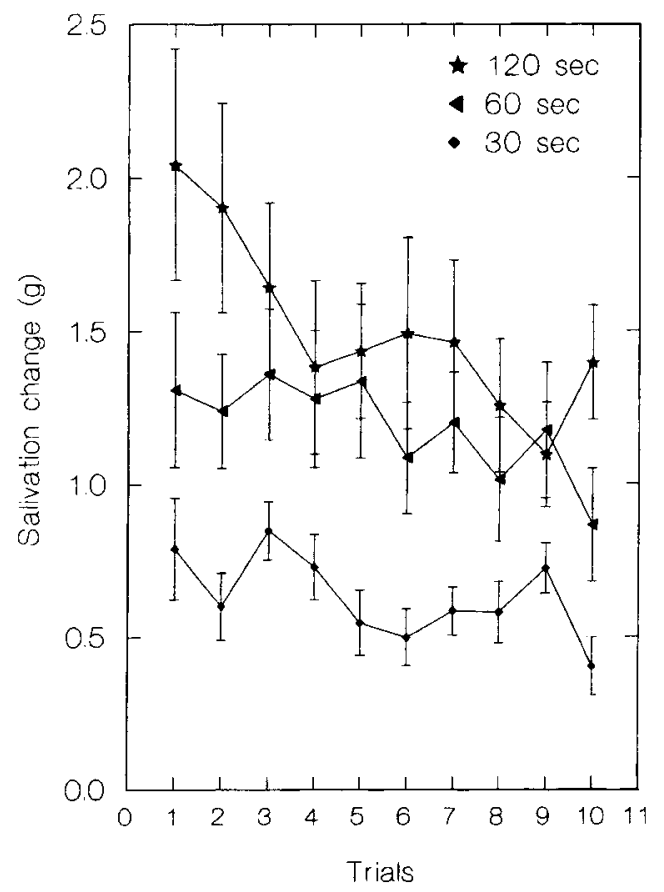

Figure 2. Changes in salivation $( \pm S E M)$ from water baseline stimulation to repeated presentation of lemon juice for $30-, 60$, and 120 -sec measurement durations.

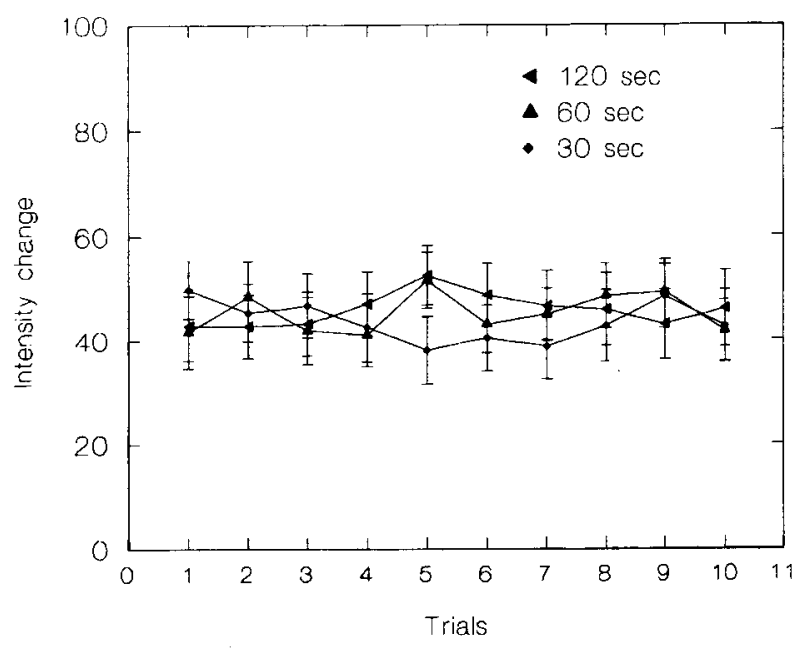

Figure 3. Changes in intensity $( \pm S E M)$ from water baseline to repeated presentation of lemon juice for 30-, 60-, and 120-sec measurement durations.

creased over trials. Similar changes in intensity ratings were observed on Trial 10 as on Trial 1 across all durations.

\section{DISCUSSION}

The results of this methodological study suggest, as expected, that the amount of saliva collected depends directly upon the duration of measurement. Within the time durations studied, the longer the stimulation and measurement interval, the more the salivation. The strong relationship between measurement duration and amount of salivation suggests that a shorter duration of measurement may discriminate among acute treatments, as does a $120-$ sec duration.

The subjects showed a general decrease in salivation across the 10 trials. Differences were observed in the rate of change, with a greater decrease for the 120 -sec than for the $30-\mathrm{sec}$ measurement duration. These differences may have been due to the larger initial response, since the shape of the habituation curve varies as a function of the magnitude of the initial response (Siddle et al., 1983). It is also possible that the longer measurement duration provides greater contact with the gustatory stimulus, providing for more stimulus processing and more habituation.

The pattern of salivary response observed to the shortduration and longer duration gustatory stimuli may be specific to the type of gustatory stimulus used. The pattern of salivation responses to more complex food cues may differ, requiring increased stimulus processing or may show a slower response in comparison with the rapid and strong reflexive responses we observed to lemon juice. In addition, salivation to single or repeated presentations of an olfactory food cue may produce a pattern of responses different from that to gustatory cues.

The absence of a consistent decrease in perceived intensity suggests that the decreases were not due to changes in the perception of the intensity of the sweetened lemon 
juice, as would have been the case if factors related to sensory fatigue, rather than habituation, were responsible for this effect. In an animal research paradigm in which monkeys were presented repeatedly with the same gustatory stimulus, there was no decrease in neuronal responding in the gustatory taste cortex, while activity in the lateral hypothalamus reliably decreased (Rolls et al., 1986; Rolls, Scott, Sienkiewicz, \& Yaxley, 1988).

No differences were observed for subjects in the groups that varied the rate of stimulus presentation keeping the intertrial interval constant versus varying the intertrial interval with the rate of stimulus presentation constant. Although research suggests that short intertrial intervals can be associated with more rapid habituation (Germana, 1969), this effect depends upon the response being measured and on the length of the interval (Siddle et al., 1983).

In summary, the results of this study suggest that duration of the measurement interval influences the amount of saliva collected in response to a single presentation of a simple gustatory stimulus, as well as the pattern of responding to repeated stimulus presentations. Shorter measurement periods are associated with smaller decreases after repeated stimulus presentations, which may influence interpretation of gustatory effects on sensory changes that influence intake. Experimental protocols that use longer measurement durations may be more sensitive to changes in patterns of response to repeated stimulation than protocols that use shorter stimulus durations.

\section{REFERENCES}

Epstein, L. H., Caggiula, A. R., Perkins, K. A., Mitchell, S. L., \& RODEFER, J. (1992). Abstinence from smoking influences habituation to food cues. Physiology \& Behavior, 52, 641-646.

Epstein, L. H., Caggivla, A. R., Rodefer, J. S., Wisniewski, L., \& MitChell, S. L. (1993). The effects of calories and taste on habituation of the human salivary response. Addictive Behaviors, 18, 179-185.

Epstein, L. H., Mitchell, S. L., \& Caggivla, A. R. (1993). The effect of subjective and physiological arousal on dishabituation of salivation. Physiology \& Behavior, 53, 593-597.

Epstein, L. H., Rodefer, J. S., Wisniewski, L., \& Caggiula, A. R. (1992). Habituation and dishabituation of human salivary response. Physiology \& Behavior, 51, 945-950.

Germana, J. (1969). Response characteristics and the orienting reflex. Journal of Experimental Psychology, 78, 610-616.

Guy-Grand, B., \& Goga, H. (1981). Conditioned salivation in obese subjects with different weight kinetics. Appetite, 2, 351-355.
Herman, C. P. (1978). Restrained eating. Psychiatric Clinics of North America, 1, 593-607.

Klajner, F., Herman, C. P., Polivy, J., \& Chabra, R. (1981). Human obesity, dieting, and anticipatory salivation to food. Physiology \& Behavior, 27, 195-198.

Legoff, D. B., Leichner, P., \& Spiegelman, M. N. ( 1988). Salivary response to olfactory food stimuli in anorexics and bulimics. Appetite, $11,15-25$.

Legoff, D. B., \& Spiegelman, M. N. (1987). Salivary response to olfactory food stimuli as a function of dietary restraint and body weight. Appetite, 8, 29-35.

Must, A., Dallal, G. E., \& Dietz, W. H. (1991). Reference data for obesity: 85 th and 95 th percentiles of body mass index (wt/ht 2 ) and triceps skinfold thickness. American Journal of Clinical Nutrition, 53, 839-846.

Navaresh, M., \& Christenen, C. M. (1982). A comparison of whole mouth resting and stimulated salivary measurement procedures. Journal of Dental Research, 6, 1158-1162.

Pangborn, R. M., Witherly, S. A., \& Jones, F. (1979). Parotid and whole-mouth secretion in response to viewing, handling, and sniffing food. Perception, 8, 339-346.

PECK, R. E. (1959). The SHP Test-an aid in the detection and measurement of depression. Archives of General Psychiatry, 1, 35-40.

Rolls, E. T., Murzi, E., Yaxley, S., Thorpe, S. J., \& Simpson, S. J. (1986). Sensory-specific satiety: Food-specific reduction in responsiveness of ventral forebrain neurons after feeding in the monkey. Brain Research, 368, 79-86.

Rolis, E. T., SCOTT, T. R., Sienkiewicz, Z. J., \& YAXLEY, S. (1988). The responsiveness of neurons in the frontal opercular gustatory cortex of the macaque monkey is independent of hunger. Journal of Physiology, 397, 1-12.

SidDle, D., STEPHENSON, D., \& SPINKS, J. A. (1983). Elicitation and habituation of the orienting response. In D. Siddle (Ed.), Orienting and habituation: Perspectives in human research (pp. 109-182). New York: Wiley.

SMithers-MulveY, S. E., \& HALl, W. G. (1992). Control of ingestion by oral habituation in rat pups. Behavioral Neuroscience, 106, 710-717.

StaATs, A. W., \& Hammond, O. W. (1972). Natural words as psychological conditioned stimuli: Food-word-elicited salivation and deprivation effects. Journal of Experimental Psychology, 96, 206-208.

WARDLE, J. (1987). Hunger and satiety: A multidimensional assessment of responses to caloric loads. Physiology \& Behavior, 40, 577-582.

WhITE, K. D. (1977). Salivation: A review and experimental investigation of major techniques. Psychophysiology, 14, 203-212.

Wisniewski, L., Epstein, L. H., \& Caggivla, A. R. (1992). The effect of food change on consumption, hedonics and salivation. Physiology \& Behavior, 52, 21-26.

Wooley, S. C., \& Wooley, O. W. (1973). Salivation to the sight and thought of food: A new measure of appetite. Psychosomatic Medicine, 35, 136-142.

(Manuscript received March 29, 1994; revision accepted for publication September 15, 1994.) 